\section{Phantom-limb pain as a perceptual correlate of cortical reorganization following arm amputation}

\author{
H. Flor*, T. Elbert $\dagger$, S. Knecht + , C. Wienbruch $\ddagger$, \\ C. Pantev $\ddagger$, N. Birbaumer $\$$, W. Larbig $\$$ E. Taub
}

\begin{abstract}
* Department of Psychology, Humboldt-University, Hausvogteiplatz 5-7, D-10117 Berlin, Germany

† Department of Psychology, University of Konstanz, Postfach 5560 , D44, D-78434 Konstanz, Germany

\$ Department of Neurology and Institute of Experimental Audiology, University of Münster, Kardinal-von-Galen-Ring 10,

D-48129 Münster, Germany

$\S$ Institute of Medical Psychology and Behavioural Neurobiology, University of Tübingen, Gartenstr. 29, D-72074 Tübingen, Germany | Department of Psychology, University of Alabama at Birmingham, Birmingham, Alabama 35294, USA
\end{abstract}

Although phantom-limb pain is a frequent consequence of the amputation of an extremity, little is known about its origin ${ }^{1-4}$. On the basis of the demonstration of substantial plasticity of the somatosensory cortex after amputation ${ }^{5}$ or somatosensory deafferentation in adult monkeys ${ }^{6}$, it has been suggested that cortical reorganization could account for some non-painful phantom-limb phenomena in amputees and that cortical reorganization has an adaptive (that is, pain-preventing) function ${ }^{2,5,7,8}$. Theoretical and empirical work on chronic back pain ${ }^{9,10}$ has revealed a positive relationship between the amount of cortical alteration and the magnitude of pain, so we predicted that cortical reorganization and phantom-limb pain should be positively related. Using non-invasive neuromagnetic imaging techniques to determine cortical reorganization in humans ${ }^{11-13}$, we report a very strong direct relationship $(r=0.93)$ between the amount of cortical reorganization and the magnitude of phantom limb pain (but not non-painful phantom phenomena) experienced after arm amputation. These data indicate that phantom-limb pain is related to, and may be a consequence of, plastic changes in primary somatosensory cortex.

A brief telephone interview was used to obtain information about the amount of phantom-limb pain in 65 upper-limb ampu- tees. This information served as the sole basis for the selection of a representative sample of 13 subjects with widely varying degrees of phantom-limb pain. The mean age of the 13 subjects was 50.1 years (s.d. $=17.2$, range $27-73 \mathrm{yr}$ ), mean post-amputation time was 24.3 years (s.d. $=19.8$, range 1 to $51 \mathrm{yr}$ ). Twelve men and one woman participated in the study. Traumatic injury in ten cases and osteosarcoma in three cases had made the amputation necessary. Cortical reorganization was determined by magnetic source imaging" using the method illustrated in Fig. 1. The subjects underwent a comprehensive neurological and psychological investigation which included detailed assessments of phantom pain and phantom sensations, stump pain and stump sensations, pre-amputation pain, telescoping (the subjective experience of the phantom limb retracting towards and often disappearing in the stump), and facial remapping (the appearance of phantom sensations upon non-painful stimulation of the face with isomorphism between facial stimulation sites and the location of phantom sensations) (Fig. 2 legend).

A large significant positive linear relationship was found between the amount of phantom-limb pain, as measured on the standardized pain-intensity scale, and the amount of cortical reorganization ( $r=0.93, P<0.0001$; Fig. 2); phantom-limb pain explained almost $85 \%$ (adjusted $r^{2}=0.83 ; \quad F(1,11)=66.89$, $P<0.0001)$ of the variance in cortical reorganization. A similar correlation was obtained for the visual analogue phantom pain scale $(r=0.85, P<0.001)$ and the adjective phantom pain scale $(r=0.86, P<0.001)$.

The mean shift in the focus of cortical responsivity to facial stimulation was $0.43 \mathrm{~cm}$ (s.d. $=0.40$, range $0.01-1.00$ ) for the five pain-free subjects, whereas the mean shift $(M)$ for the eight subjects with phantom-limb pain was almost five times as large $(M=2.05 \mathrm{~cm}, \quad$ s.d. $=1.08$, range $0.52-3.86 ; \quad F(1,11)=9.94$, $P<0.01$ ) (Fig. 3). None of the measures for stump pain, nonpainful stump sensations or non-painful phantom sensations showed a significant relationship to amount of cortical reorganization. The presence, frequency and intensity of telescoping, or the length of the telescoped phantom, as well as reports of facial remapping (which was present in 4 of the 13 subjects) were unrelated to cortical reorganization. None of the five patients without phantom pain reported the experience of pre-amputation pain. However, half of the eight patients with phantom pain had experienced pain before the amputation (three due to traumatic injury, and one due to osteosarcoma); the other half 
FIG. 1 Illustration of the method used to determine the amount of cortical reorganization. The centres of the magnetic responses to stimulation on the face and digits are superimposed onto a magnetic resonance image of an individual subject.

METHODS. In all subjects at least the following four sites were stimulated by using light superficial pressure applied via a pneumatic stimulator: (1) the first and (2) the fifth digits of the (intact) hand; (3) the chin below the left corner of the lip (first five subjects) or the lower lip near the left corner of the mouth (eight subjects) and (4) the chin below the right corner of the lips (first five subjects) or the lower lip near the right corner of the mouth (eight subjects). The recording from the lip was found to yield a much better cortical response than the recording from the chin and was therefore preferred in the latter series of recordings. During these four conditions, the sensor array was positioned over the hemisphere contralateral to the site of stimulation. At each site 1,000 stimuli were delivered at an average stimulation rate of $0.5 \mathrm{~Hz}$ (interval between stimulus onsets: $500 \pm 50 \mathrm{~ms}$ ). The sequence of sites at which stimuli were presented was varied according to a fixed irregular order across subjects. After each train of 1,000 stimuli, the subjects indicated the primary and secondary (if any) location of the perceived stimulations, as well as its quality and intensity. Using a BTi neuromagnetometer, magnetic fields were recorded from 37 locations over a circular concave area $(14.4 \mathrm{~cm}$ diameter) above the parietotemporal cortex contralateral to the site of the stimulation. Recordings were carried out in a magnetically shielded room. Subjects lay in a lateral position with their whole body supported by vacuum cushions. The MEG was sampled at a rate of $520.5 \mathrm{~Hz}$. The evoked magnetic responses from each stimulation site were averaged (from -100 to $+250 \mathrm{~ms}$ ) and digitally filtered with a bandpass of 0.01 to $100 \mathrm{~Hz}$. To exclude artefacts, a response was omitted from the average if its range exceeded $2 \mathrm{pT}$ in any of the MEG channels. For each magnetic field distribution, a single equivalent current dipole (ECD) model (best-fitting local sphere) was fitted within the latency range from 35 to $75 \mathrm{~ms}$. From the points with a goodnessof-fit larger than 0.95 and a confidence volume smaller than $300 \mathrm{~mm}^{3}$, the region with the maximal field power (measured as root-mean-square across channels) was selected. To illustrate the measure of reorganiza-

had never experienced pre-amputation pain (Yates-corrected $\chi^{2}$, NS). The overall correlation between pre-amputation pain and phantom pain was thus only moderate and not significant. There was a trend towards a significant relationship between the amount of cortical reorganization and pre-amputation pain $(r=$ $0.51, F(1,11)=3.91, P=0.07)$ which was entirely due to the positive though nonsignificant relationship of phantom-limb pain and pre-amputation pain (partial correlation of reorganization and pre-amputation pain with phantom-limb pain removed: $r=$ -0.132 ). Age of patients at time of testing, age at time of amputation, and time elapsed since the amputation were unrelated to both phantom-limb pain and cortical reorganization (all $P$ values NS). A final stepwise regression analysis, with all pain, sensation, demographic and clinical variables entered to predict the amount of cortical reorganization, yielded a single predictor: amount of phantom-limb pain experienced by the subjects.

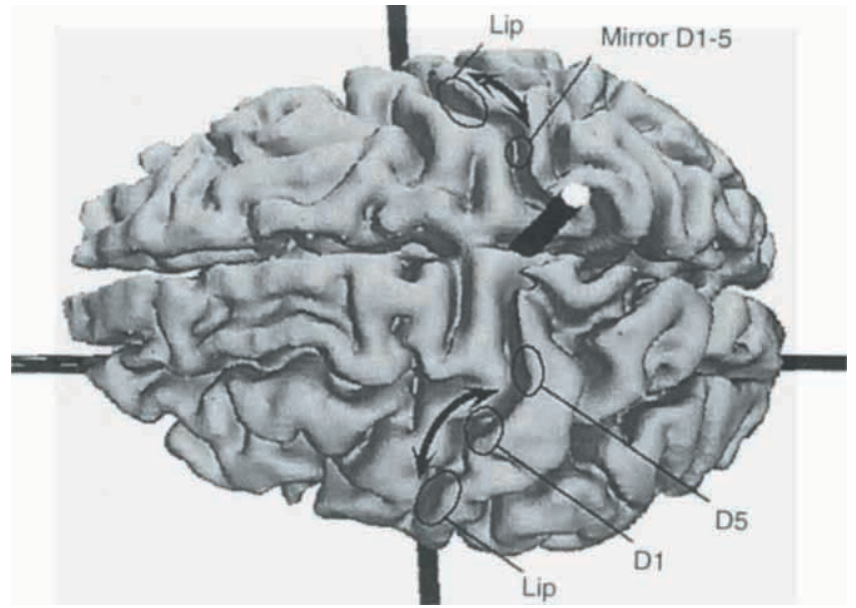

tion used, these ECD locatioris were mapped onto the cortical surface of area $3 \mathrm{~b}$, which was reconstructed from a magnetic resonance image scan as described ${ }^{20}$. Distances between locations along the curved surface of the somatosensory map can be represented by the distance in the three cortical dimensions (black rods). The arrows connecting the cortical representation of the lip and (1) the midpoint between digits 1 and 5 on the intact side (lower arrow) and (2) the midpoint between the mirror images of the first and fifth digit and the lip on the amputated side (upper arrow), represent the cortical-distance measure used. The mirror images of digits 1 and 5 were obtained by projecting the centres of magnetic activity on the intact side across the midsagittal plane onto the opposite hemisphere (representing the amputation side of the body). To obtain an estimate of the extent of the reorganization that had occurred, a comparison was made of the difference in the two distance measures (that is, the mean coronal shift in the dipole of the amputation-side face area relative to the fingers).

The results of this study reveal a strong positive relationship between the amount of cortical reorganization occurring after unilateral upper-extremity amputation and the intensity of phantom pain. The present findings suggest the intriguing possibility that the shift of the cortical map following amputation might be a potential neurophysiological basis of phantom-limb pain. The result contradicts current influential theoretical analyses of phantom-limb pain ${ }^{2.5}$, in which an inverse relationship between painful phantom-limb phenomena and cortical reorganization was postulated, but is consistent with Melzack's assumption of a "neuromatrix' underlying phantom-limb pain. The involvement of cortical reorganization in phantom-limb pain is not inconsistent with the extensive evidence that peripheral mechanisms also contribute to phantom pain ${ }^{1,2}$. The remodelling of the functional architecture of the cortex in response to nervous-system damage could serve as an adaptive compensatory mechanism by restor-
FlG. 2 Amount of cortical reorganization (in $\mathrm{cm}$ ) for each subject plotted against intensity of phantom-limb pain, as measured with the Multidimensional Pain Inventory.

METHODS. Phantom-limb and stump pain were assessed by three methods: (1) the pain intensity scale of the West Haven-Yale Multidimensional Pain Inventory ${ }^{21.22}$, a reliable and valid measure of the amount of pain experienced, administered separately for phantom and stump pain; (2) a phantom-and-stump phenomena interview that included the pain experience scale (Schmerzempfindungsskala ${ }^{23}$ ) consisting of 24 pain adjectives derived from the McGill pain questionnaire ${ }^{24}$. Each of these 24 descriptors was scored on a 4-point scale according to the extent to which it accurately described the subjects' pain experience. The scale was administered separately for phantom-limb pain, stump pain, and pre-amputation pain; (3) a $10-\mathrm{cm}$ visual analogue scale with the endpoints 'no pain' and 'unbearable pain'. Non-painful phantom phenomena (such as telescoping) and nonpainful stump sensations (such as itching, pressure) were measured in the phantom-and-stump phenomena interview. The presence of facial remapping was assessed by probing the surface of the entire body with a cotton-wool bud while the patient indicated the presence of phantom sensations ${ }^{8,11}$.

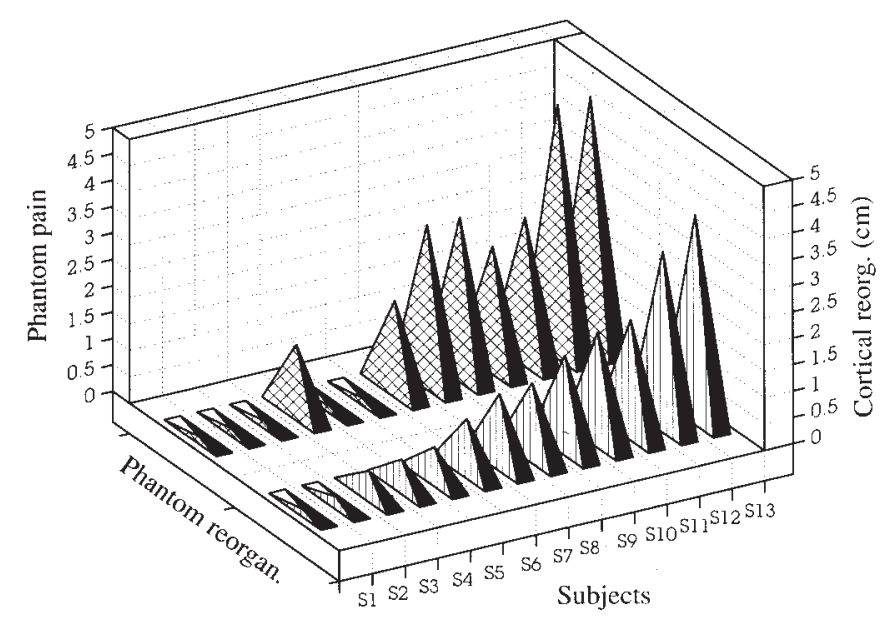




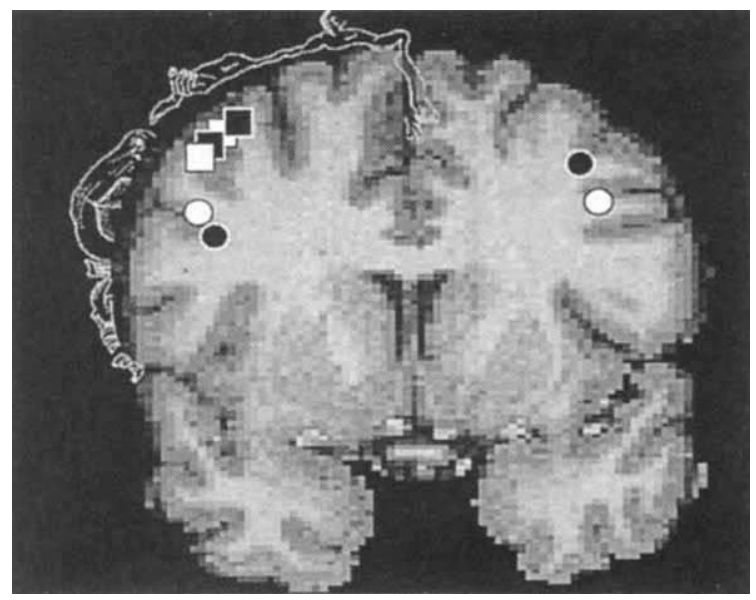

FIG. 3 A representative subject with intense phantom-limb pain (black symbols with white surround) and one subject who experienced no phantom-limb pain (white symbols with black surround) were selected from the patient sample. Squares represent the ECD locations of the digits, circles represent the location of the lip projected onto a coronal section of the brain. Hemisphere contralateral to intact side (left) and amputation side (right). A pronounced hemispheric asymmetry in the location of the lips can be observed in the phantom-pain patient.

ing activity in a zone deprived of its afferent input and could provide a basis for recovery of function. In patients with phantom-limb pain, this mechanism would appear to have become maladaptive, possibly related to a lasting hyperexcitability of nociceptive pathways induced by previous pain ${ }^{14}$, imbalance of nociceptive and non-nociceptive inputs after deafferentation ${ }^{15}$, or similar mechanisms. The reorganization observed in some subjects with phantom-limb pain (exceeding $3 \mathrm{~cm}$ in two subjects) probably encompasses the full extent of the cortical area $3 \mathrm{~b}$ representing the hand and lower arm area before amputation. It is possible that reorganization may have occurred in additional areas of SI as well as SII, which would not have been assessed by our methods. It has been suggested ${ }^{5.16}$ that the reorganization could be due to synaptic remodelling within the arborization zones of thalamocortical neurons projecting to areas of somatosensory cortex that had retained their normal sources of input and which were adjacent to the affected cortical areas that had lost their afferents (cortical face or head areas). This mechanism, however, can account for only a small fraction of the full topographic extent of the massive reorganization in the present study. To what extent chronically altered afferent inputs produced by sprouting ${ }^{17}$, increase in synaptic strength ${ }^{18}$ or unmasking of intracortical connections ${ }^{19}$ contribute to the reorganization observed here needs to be investigated further. In addition, determination of behavioural methods or pharmacological agents affecting cortical reorganization might eventually be effective in relieving phantom-limb pain.

Received 14 February; accepted 29 March 1995.

1. Jensen, T. S. \& Rasmussen, P. in Textbook of Pain (eds Wall, P. \& Melzack, R. A.) 651666 (Churchill-Livingstone, Edinburgh, 1994).

2. Katz, J. Can. J. Psychiat. 37, 282-291 (1992)

3. Melzack, R. A. Can. Psychol. 30, 1-16 (1989)

4. Sherman, R. A., Arena, J. C., Sherman, C. J. \& Ernst, J. C. Biof Self-Regul. 14, 267-280 (1989).

5. Merzenich, M. et. al. J. comp. Neurol. 224, 591-605 (1984).

6. Pons, T. et al. Science 252, 1857-1860 (1991).

7. Ramachandran, V., Rogers-Ramachandran, D. \& Stewart, M. Science 258, 1159-1160 (1992).

8. Ramachandran, V., Stewart, M. \& Rogers Ramachandran, D. Neuroreport 3, 583-586 (1992).

9. Fior, H. \& Birbaumer, N. Am. Pain Soc. J. 3, 118-127 (1994).

10. Flor, H. et al. in Recent Advances in Biomagnetism (eds Deecke, L., Baumgartner, C., Stroink, G. \& Wiltiamson, S. J.) (Elsevier, Amsterdam: in the press).

11. Elbert, T. et at. Neuroreport 5, 2593-2597 (1994).

12. Yang, T. et al. Neuroreport 5, 701-704 (1994).

13. Yang, T. et al. Nature 368, 592-593 (1994).
14. Willis, W. D. Jr in Proc. 7th Worid Congr. on Pain leds Gebhart, G. F., Hammond. D. L. \& Jensen, T. S.) 301-324 (IASP, Seattle, 1994).

15. Pain and Central Nervous System Disease (ed. Casey, K. L.) (Raven. New York 1991).

16. Lund, J. T., Sun, G. D. \& Lamarre, Y. Science 265, 546-548 (1994).

17. Darian-Smith, C. \& Gibert, C. D. Nature $\mathbf{3 6 8}, 737-740(1994)$

18. Gilbert, C. D. Curr. Opin. Neurobiol. 3, 100-103 (1993).

19. Calford, M. B. \& Tweedale, R. Proc. R. Soc. B. 243, 269-275 (1991).

20. Lütkennöner, B. et al. Brain Topography (in the press).

21. Kerns, R. D., Turk, D. C. \& Rudy, T. E. Pain 23, 345-356 (1985)

22. Flor, H., Rudy, T. E., Birbaumer, N., Streit, B. \& Schugens, M. M. Der Schmerz 4, 82-87 (1990).

23. Geissner, E. Die Schmerzempfindungsskala (Beltz, Weinheim, in the press)

24. Melzack, R. A. Pain 1, 277-299 (1975).

ACKNOWLEDGEMENTS. This work was supported by grants from the Deutsche Forschungsgemeinschaft. 